\title{
Pediatric Ovarian Torsion and its Recurrence: A Multicenter Study
}

Mirko Bertozzi MD ${ }^{1, *}$, Ciro Esposito MD, $\mathrm{PhD}^{2}$, Claudio Vella $\mathrm{MD}^{3}$, Vito Briganti MD ${ }^{4}$, Nicola Zampieri MD ${ }^{5}$, Daniela Codrich MD ${ }^{6}$, Michele Ubertazzi MD ${ }^{7}$, Alessandro Trucchi $\mathrm{MD}^{8}$, Elisa Magrini MD, $\mathrm{PhD}^{1}$, Sonia Battaglia MD ${ }^{9}$, Vittorio Bini $\mathrm{BSc}^{10}$, Maria Luisa Conighi MD ${ }^{11}$, Caterina Gulia $\mathrm{MD}^{4}$, Alessandra Farina $\mathrm{MD}^{2}$, Francesco Saverio Camoglio MD, $\mathrm{PhD}^{5}$, Waifro Rigamonti MD, PhD ${ }^{12}$, Piergiorgio Gamba MD, $\mathrm{PhD}^{9}$, Giovanna Riccipetitoni $\mathrm{MD}^{3}$, Salvatore Fabio Chiarenza MD ${ }^{11}$, Alessandro Inserra $\mathrm{MD}^{8}$, Antonino Appignani MD, $\mathrm{PhD}^{1}$

\footnotetext{
${ }^{1}$ S.C. di Clinica Chirurgica Pediatrica, Università degli Studi di Perugia, Ospedale S. Maria della Misericordia, Perugia, Italy

${ }^{2}$ Department of Translational Medical Sciences, Federico II University of Naples, Naples, Italy

${ }^{3}$ S.C. di Chirurgia Pediatrica, Ospedale dei Bambini “V. Buzzi,” Milano, Italy

${ }^{4}$ U.O.C. di Chirurgia e Urologia Pediatrica Azienda Ospedaliera S. Camillo Forlanini, Roma, Italy

${ }^{5}$ Pediatric Surgical Unit, Department of Surgical Sciences, G.B. Rossi Hospital, Verona, Italy

${ }^{6}$ Institute for Maternal and Child Health, IRCCS “Burlo Garofolo" Via dell'Istria, Trieste, Italy

${ }^{7}$ U.O. di Chirurgia Pediatrica, Azienda Ospedaliero, Universitaria di Sassari Viale S. Pietro, Sassari, Italy

${ }^{8}$ U.O.C. Chirurgia Generale e Toracica Ospedale Pediatrico Bambino Gesù, Rome, Italy

${ }^{9}$ Pediatric Surgery, Department of Woman and Child Health, University Hospital, Padua, Italy

${ }^{10}$ Department of Medicine, Section of Internal Medicine and Endocrine and Metabolic Sciences, University of Perugia, Perugia, Italy

${ }^{11}$ U.O di Chirurgia Pediatrica, Ospedale S. Bortolo, Vicenza, Italy

${ }^{12}$ Institute for Maternal and Child Health IRCCS “Burlo Garofolo," University of Trieste, Trieste, Italy
}

\section{A B S T R A C T}

Study Objective: To report results of a retrospective multicentric Italian survey concerning the management of pediatric ovarian torsion (OT) and its recurrence.

Design: Multicenter retrospective cohort study.

Setting: Italian Units of Pediatric Surgery.

Participants: Participants were female aged 1-14 years of age with surgically diagnosed OT between 2004 and 2014.

Interventions: Adnexal detorsion, adnexectomy, mass excision using laparoscopy or laparotomy. Different kinds of oophoropexy (OPY) for OT or recurrence, respectively.

Main Outcome Measures: A total of 124 questionnaires were returned and analyzed to understand the current management of pediatric OT and its recurrence. The questionnaires concerned patient age, presence of menarche, OT site, presence and type of mass, performed procedure, OPY technique adopted, intra- and postoperative complications, recurrence and site, procedure performed for recurrence, OPY technique for recurrence, and 1 year follow-up of detorsed ovaries.

Results: Mean age at surgery was $9.79 \pm 3.54$ years. Performed procedures were open adnexectomy (52 of 125; $41.6 \%$ ), laparoscopic adnexectomy ( 25 of $125 ; 20 \%$ ), open detorsion (10 of 125; 8\%), and laparoscopic detorsion (38 of $125 ; 30.4 \%$ ). Recurrence occurred in 15 of 125 cases $(12 \%)$ and resulted as significant $(P=.012)$ if associated with a normal ovary at the first episode of torsion. Recurrence occurred only in 1 of 19 cases after OPY (5.2\%). Ultrasonographic results of detorsed ovaries were not significant whether an OPY was performed or $\operatorname{not}(P=1.00)$.

Conclusion: Unfortunately, oophorectomy and open technique are still widely adopted even if not advised. Recurrence is not rare and the risk is greater in patients without ovarian masses. OPY does not adversely affect ultrasonographic results at 1 year. When possible OPY should be performed at the first episode of OT.

Key Words: Ovarian torsion, Recurrence, Oophoropexy, Children

\section{Introduction}

Ovarian torsion (OT) is a rare condition in pediatric patients. Overall, approximately $15 \%$ of cases occur during infancy and childhood ${ }^{1}$ and this percentage is greater if adolescent and teenage patients are included. ${ }^{2}$

\footnotetext{
The authors indicate no conflicts of interest.

* Address correspondence to: Mirko Bertozzi, MD, S.C. di Clinica Chirurgica Pediatrica, Università degli Studi di Perugia, Ospedale S. Maria della Misericordia, Loc. S. Andrea delle Fratte, 06100 Perugia, Italy; Phone: +39-0755786451

E-mail address: mirkobertozzi@hotmail.com (M. Bertozzi).
}

Large series from pediatric centers report an incidence between 0.3 and 3.5 cases per year. ${ }^{1,3-9}$

In pediatric patients the torsion of normal ovaries ranges from $16 \%$ to $49 \%$ and the remaining percentage is associated with the presence of masses. . $^{3,10-12}$

The etiology of OT in the absence of cysts or masses is more obscure. Contributing factors could be the hypermobility caused by an elongated ovarian ligament or a hyperlax mesosalpinx or meso-ovarium. ${ }^{13}$

In the case of OT, adnexectomy appears largely abandoned for a conservative management also in the case of a necrotic-appearing ovary. ${ }^{14,15}$ 
Indeed, recent literature suggests that standard care for OT is detorsion without removal of the fallopian tube/ovary and excision or aspiration of the cause of the torsion. $^{2}$

OT recurrence is rarely reported and different surgical techniques to limit ovarian mobility, ${ }^{16-20}$ also called oophoropexy (OPY), have been described.

There are concerns that OPY might damage the ovary or distort the relationship between ovary follicles and the fallopian tubes. $^{2}$

Indeed, alteration of pelvic anatomy during OPY might lead to tubal occlusion and impaired tube-ovary interaction. ${ }^{21}$ Nevertheless, there is no evidence in the literature that OPY decreases fertility. ${ }^{2}$

Here we report results of a multicenter Italian survey of a 10-year retrospective study concerning the treatment of OT and its recurrence in children. This report describes the management of 10 different centers in the case of OT and OT recurrence as well as results at 1 year follow-up of the preserved ovaries, to describe the current management of OT.

\section{Materials and Methods}

Data collection ranged from January 2004 to January 2014 and was carried out by a questionnaire sent to 10 Italian centers of pediatric surgery. A total of 124 questionnaires were returned and analyzed. The information obtained was: (1) patient age; (2) presence of menarche; (3) site of torsion; (4) presence of mass in the torsed ovary; (5) type of mass; (6) type of procedure performed; (7) associated procedures; (8) if an OPY was performed after the first episode of torsion, the site and the technique adopted (open or laparoscopic); (9) the OPY technique; (10) complications and type of complication that occurred during this procedure; (11) postoperative complications; (12) recurrence and site of recurrence after first procedure for torsed ovary; (13) how many times after OT recurrence occurred; (14) procedure performed for the OT recurrence; (15) whether an open or laparoscopic OPY was performed for the recurrence and the adopted technique; and (16) follow-up at 12 months of detorsed ovaries.

Either $\chi^{2}$ or Fisher exact test were used to analyze categorical data and odds ratios in the significant tests were also calculated. All calculations were carried out with IBMSPSS version 22.0 (IBM Corp, Armonk, NY) and a 2-sided $P<.05$ was considered significant.

There were no criteria for patient selection except the presence of OT and selected age from 1 to 14 years.

Its retrospective design represents the major limitation of this study.

\section{Results}

The mean age at the time of surgery was $9.79 \pm 3.54$ (range, 2-14) years. Eighty-four of 124 patients were premenarchal (67.7\%) and 40 of 124 were postmenarche (32.3\%). Torsion involved the right ovary in 82 of 125 cases (66.1\%) and left OT was reported in 41 of 125 cases (33\%). In 1 of 125 patients the OT was bilateral and synchronous (0.9\%).

In 59 of 125 cases (47.2\%; 39 right, 20 left) the OT was caused by the presence of a mass; the other 66 of 125 torsed
Table 1

Types of Mass Causing Ovarian Torsion

\begin{tabular}{lcr}
\hline \multicolumn{1}{c}{ Type of Mass } & $\mathrm{n}$ & $\%$ \\
\hline Teratoma & 19 & 32.2 \\
Simple cyst & 21 & 35.6 \\
Hemorrhagic cyst & 11 & 18.6 \\
Dermoid cyst & 3 & 5.1 \\
Paramesonephric cyst & 2 & 3.4 \\
Fibrous cystoadenoma & 1 & 1.7 \\
Serous cystoadenoma & 1 & 1.7 \\
Granulosa cell tumour & 1 & 1.7 \\
\hline
\end{tabular}

ovaries (52.8\%; 44 right, 22 left) were normal. Table 1 shows the types of mass that caused the OT.

The performed procedures were open adnexectomy in 52 of 125 cases (41.6\%), laparoscopic adnexectomy in 25 of 125 cases (20\%), open detorsion in 10 of 125 cases ( $8 \%$ ), and laparoscopic detorsion in 38 of 125 cases (30.4\%).

An open approach was started in 50 of 124 patients (40.3\%) and a laparoscopic procedure in 74 of 124 cases (59.7\%), but conversion to open surgery occurred in 11 of 74 cases (14.9\%). In 9 of 11 cases (81.8\%) conversion to open surgery occurred for the presence and treatment of an ovarian mass and in 2 of 11 cases (18.2\%) conversion was done because of technical problems.

OPY was performed at the first episode of OT in 8 cases. In 6 cases the OPY was performed ipsilaterally after detorsion of the twisted ovary (2 laparoscopic, 4 open) and in 2 cases the OPY was performed in the contralateral ovary after adnexectomy (1 laparoscopic and 1 open).

In 11 cases OPY was performed after an OT recurrence. In 5 cases recurrence occurred after a laparoscopic detorsion and it was always ipsilateral. In 6 cases the recurrence occurred contralaterally after a previous adnexectomy performed in 5 cases via open technique and in 1 case laparoscopically.

OT recurrence occurred in 15 of 125 cases (12\%) after a median period of 14.9 months after previous intervention for OT (range, 5-72 months; mean, $21 \pm 16.5$ months).

Recurrences occurred ipsilaterally in 8 cases of previous laparoscopically detorsed ovary. In 1 of these a cyst marsupialization was also performed.

Recurrences occurred contralaterally in 7 patients, in particular in 5 cases of previous open adnexectomy, in 1 case of previous laparoscopic adnexectomy, and in another laparoscopic adnexectomy associated with a contralateral cyst marsupialization.

Procedures for ipsilateral recurrence were 4 open detorsions, 1 laparoscopic detorsion, and 3 laparoscopic adnexectomies. Procedures performed for contralateral recurrence were 4 open detorsions and 3 laparoscopic detorsions.

In all cases of recurrent OT an OPY was performed except in 3 cases of adnexectomy and in 1 case of simple detorsion.

In 13 cases recurrence occurred in previously detorsed ovaries without a mass and only in 2 cases it occurred in ovaries with a mass $(P=.012)$. In 1 of these cases recurrence occurred even if an OPY had been performed at the first intervention of OT.

OT recurrence was statistically significant if associated with a normal ovary at the first episode of torsion compared with those with a mass (odds ratio, 0.143 ; 95\% 
Table 2

Comparison between the Presence of a Mass or Not at the First Episode of Torsion in Recurrent Torsed Ovary

\begin{tabular}{cccc}
\hline & \multicolumn{2}{c}{ Recurrence } & Total \\
\cline { 2 - 3 } & No & Yes & \\
\hline $\begin{array}{c}\text { Torsed ovary } \\
\text { Normal } \\
n\end{array}$ & 53 & & \\
$\%$ & 80.3 & $13^{*}$ & 66 \\
With mass & & $19.7^{*}$ & 100.0 \\
$n$ & 57 & 2 & 59 \\
$\%$ & 96.6 & 3.4 & 100.0 \\
$\begin{array}{c}\text { Total } \\
n\end{array}$ & 110 & 15 & 125 \\
$\%$ & 88.0 & 12.0 & 100.0 \\
\hline
\end{tabular}

* The specified comparison was statistically significant $(P=.012)$

confidence interval, 0.031-0.664; $P=.012$; Table 2). Indeed, the risk of recurrence of OT is $6.991(1 / 0.143)$ greater in patients without the presence of ovarian masses at the first episode of torsion compared with those with ovarian masses.

Furthermore, recurrence was not statistically related to presence of menarche at the first episode of torsion $(P=.156)$.

In all cases of recurrent OT when the surgical choice was detorsion, an OPY was performed except for 1 patient (11 of 12; 91.6\%). Recurrence occurred only in 1 case among fixed ovaries ( 1 of $19 ; 5.2 \%$ ).

Follow-up at 1 year after detorsion of twisted ovaries was obtained using color Doppler ultrasonography in 42 of 48 patients (87.5\%). Six patients were lost to follow-up. After 1year follow-up 36 detorsed ovaries were well vascularized with normal parenchyma whereas 6 ovaries were vascularized but hypoplastic and/or with dishomogenous parenchyma.

Follow-up results of detorsed ovaries were not statistically significant $(P=1.00)$ whether an OPY was performed or not (Table 3$)$.

\section{Discussion}

OT is an uncommon event in the pediatric age group. ${ }^{22}$ Approximately half of these cases involves an ovarian mass. ${ }^{3,10-12,23,24}$ However, several case series with torsion

Table 3

Comparison of Color Doppler Ultrasonographic Results if an Oophoropexy Was Performed or Not after Detorsion

\begin{tabular}{cccc}
\hline & \multicolumn{2}{c}{$\begin{array}{c}\text { Color Doppler Ultrasonographic Results } \\
\text { at 1 Year after Treatment }\end{array}$} & Total \\
\cline { 2 - 3 } & $\begin{array}{c}\text { Well Vascularized } \\
\text { with Normal } \\
\text { Parenchyma }\end{array}$ & $\begin{array}{c}\text { Vascularized But Hypoplastic } \\
\text { and/or with Dishomogenous } \\
\text { Parenchyma }\end{array}$ & \\
\cline { 1 - 3 } & & & \\
Oophoropexy & 27 & 5 & 32 \\
n & 84.4 & 15.6 & 100.0 \\
Yes & 9 & 1 & 10 \\
n & 90.0 & 10.0 & 100.0 \\
Total & 36 & 6 & 42 \\
n & 85.7 & 14.3 & 100.0 \\
\hline$\%$ & & & \\
\hline
\end{tabular}

of normal premenarchal ovaries have been reported in the literature. ${ }^{7,10,23}$

In our multicenter study results confirmed data reported in the literature because in $47.2 \%$ of the cases the OT was caused by the presence of a mass and in $52.8 \%$ there was a normal ovary.

Eighty-four of 124 patients were in the premenarchal period (67.7\%) and 40 of 124 were postmenarche (32.3\%). Indeed, in an age ranging between 1 and 14 years most OTs occurred in premenarchal girls.

The excessive mobility of the adnexa due to an abnormally long fallopian tube, mesosalpinx, or meso-ovarium are the reported theories proposed to explain OT in a normal ovary and possible mechanisms that can cause torsion are abrupt changes in intra-abdominal pressure with vomiting and coughing, adnexal venous congestion due to constipation, sigmoid distention, or premenarchal hormonal activity and sudden acceleration/deceleration movements in the presence of a small infantile uterus and a relatively large ovary. ${ }^{7,15,25,26}$

OT is more common on the right side than on the left side. ${ }^{26,27}$ This incidence might be explained presumably because the presence of the sigmoid colon is responsible for reduced mobility of the left adnexa. ${ }^{28}$ In our experience torsion involved the right ovary in $66.1 \%$ of cases ( 82 of 125 ) and involvement of the left ovary was reported in 33\% cases (41 of 125). Bilateral synchronous torsion was exceptional (0.9\%; 1 of 125$)$.

Nowadays the literature reports that OTs in children are managed conservatively with simple detorsion ${ }^{13,29,30}$ and laparoscopy appears to be the most diffused technique to approach this kind of condition. Nevertheless, our collected data suggest that in the case of OT, adnexectomy is still the most performed procedure (61.6\%; 77 of 125$)$ compared with conservative management (38.4\%; 48 of 125).

The performed procedures were open adnexectomy in 52 of 125 cases (41.6\%), laparoscopic adnexectomy in 25 of 125 cases (20\%), open detorsion in 10 of 125 cases (8\%), and laparoscopic detorsion in 38 of 125 cases (30.4\%). Furthermore, the laparoscopic approach to OT was started in 74 of 124 cases (59.7\%) but conversion to open surgery occurred in 11 of 74 cases (14.9\%). Reasons for conversion to open surgery were in $81.8 \%$ ( 9 of 11 ) the presence and treatment of an ovarian mass and in $18.2 \%$ ( 2 of 11 ) conversion was performed because of technical problems. This could be because OT is an emergency; the surgical approach and/or conversion also depends on the laparoscopic experience of the surgeon involved in this kind of emergency.

In the pediatric literature recurrent OT after detorsion is rarely described. ${ }^{3,31}$ Nevertheless, in this 10 -year multicenter retrospective study OT recurrence occurred in 15 of 125 cases (12\%).

This study shows a minor frequency of recurrent OT if an open detorsion is performed at the first episode of torsion ( 0 of 9) compared with laparoscopic detorsion (8 of $31 ; 0 \%$ vs $20.5 \%)$ even if not statistically significant $(P=.321)$. These data could be explained by a major role played by adherences formation after an open detorsion that could avoid a recurrence of torsion, compared with a laparoscopic approach. In fact results highlight that in the case of previous open 
adnexectomy contralateral recurrence occurred in $9.6 \%$ vs $8 \%$ ( 5 of 52 vs 2 of 25) compared with laparoscopic adnexectomy $(P=1.00)$.

Recurrence occurred in previously detorsed ovaries without a mass in $86.6 \%$ (13 of 15 ) of the cases and only in $13.3 \%$ ( 2 of 15 ) of the ovaries with a mass $(P<.05)$. In 1 of these cases recurrence occurred even if an OPY was performed at the first intervention of OT.

OT recurrence was more common among girls without the presence of ovarian masses at the first episode of torsion compared with those who presented a mass. In statistical analysis the estimated risk of recurrence of OT is generally approximately 7 times greater in patients without ovarian masses compared with those with ovarian masses.

Recurrence occurred after a median period of 12 months after previous intervention for OT. Contrary to what Smorgick et al claim, ${ }^{32}$ recurrences were not statistically related to the presence of menarche at the first episode of torsion $(P=.156)$. Probably we obtained different results because the age range of our analyzed patients was younger (114 years) compared with that of the previously mentioned authors (1-18 years).

Whether to perform OPY after OT or not is a debated topic. OPY could be performed in the ipsilateral torsed ovary after its detorsion or in the contralateral ovary if an ovariectomy of the torsed ovary is performed. The literature reports different opinions about whether and when OPY should be performed including the type of surgical procedure and whether both adnexa should be fixed or not.

The major issue is whether OPY should be performed and the type of OPY adopted because some kinds of OPY do not exclude recurrence. ${ }^{12,33-36}$

In fact, in our study we also had 1 case of recurrence after OPY using the plication of the utero-ovarian ligament.

A recent review of the pediatric literature reported that the first common approach to OT is only detorsion without $\mathrm{OPY}^{31}$ even if in adults in the case of torsion of a normal ovary, simple detorsion without OPY can predispose patients to recurrence at a rate up to $63.6 \%{ }^{37}$ Nevertheless, our multicenter study showed that OPY was rarely performed at the first episode of OT ( 8 of 125 cases; 6.4\%). In 6 cases the OPY was performed ipsilaterally after detorsion of the twisted ovary (2 laparoscopic detorsions and 4 open detorsions). In 2 cases OPY was performed in the contralateral ovary after adnexectomy (1 laparoscopic adnexectomy and 1 open adnexectomy). OPY techniques were adopted in 6 cases with a lateral side wall fixation of the ovary, in 1 case with a fixation to the posterior uterine wall, and in the last case with a utero-ovarian ligament plication.

On the contrary, OPY after OT recurrence is a widely diffused choice. In this retrospective study in all cases of recurrent OT when the surgical choice was a detorsion, an OPY was performed (73.3\%; 11 of 15) except in 1 of 15 patients (6.7\%) and in 3 of 15 cases of adnexectomy (20\%).

In these last cases OPY was performed in 8 cases using an open technique whereas laparoscopic detorsion was performed in 3 cases.

Different types of OPY techniques are described in the adult and pediatric literature such as the pelvic lateral sidewall fixation, the plication of the ovarian ligament, ovarian fixation to the ovaric fossa level, suturing the ovary to the posterior wall of the uterus, or the fixation of the ovary to the round ligament. ${ }^{16-20}$

In this experience of OPY techniques for recurrent torsion in 7 of 11 cases (63.6\%) the ovary was fixed to the lateral pelvic sidewall, in 2 of 11 cases (18.2\%) it was fixed to the ovarian fossa, in 1 of 11 case (9.1\%) to the posterior uterine wall, and in the last case of 11 it was fixed to the round ligament (9.1\%).

The most frequent OPY technique adopted was the pelvic lateral sidewall fixation, even if this technique does not exclude recurrence, ${ }^{38}$ as well as plication of the ovarian ligament, ${ }^{36}$ as reported also in 1 of our cases. Other techniques such as ovarian fixation to the level of the ovaric fossa, suturing the ovary to the posterior wall of the uterus, and fixation of the ovary to the round ligament, are performed with less frequency.

According to most authors it seems reasonable to perform a bilateral procedure only in patients with asynchronous bilateral torsion of normal adnexa. ${ }^{3}$ Fixation does not necessarily eliminate the risk of retorsion. Although the exact numbers are not known, Tsafrir et al reported a 9.5\% rate of retorsion after adnexal fixation. ${ }^{39}$

Nevertheless, the best technique for ovarian fixation to eliminate the risk of recurrence has yet to be determined and the choice of OPY technique should be individualized. ${ }^{40}$

Laparoscopic OPY involving fixation of the ovary to neighboring structures needs advanced laparoscopic tissue handling and suturing skills ${ }^{36}$ and we had the same impression from our results because in a total of 19 performed oophoropexies, the procedure was the open technique in 11 of 19 cases (57.9\%) and only in 8 of 19 cases (42.1\%) using a laparoscopic technique.

Color Doppler ultrasonography follow-up at 1 year after detorsion of twisted ovaries was obtained in 42 of 48 patients. After 1-year follow-up 36 of 42 detorsed ovaries were vascularized and with normal size (85.7\%) whereas 6 of 42 ovaries were vascularized but hypoplastic and/or with dishomogenous parenchyma (14.3\%). Follow-up results of detorsed ovaries were not statistically significant $(P=1.00)$ whether an OPY was performed or not.

In conclusion, OT is more common in premenarchal girls (67.7\%; 84 of 124). Bilateral synchronous OT is exceptional ( $0.8 \%$; 1 of 124 patients). OT recurrence in the pediatric age group is not so rare $(12 \% ; 15$ of 125$)$ and the risk is approximately 7 times greater in patients without ovarian masses at the first episode of torsion.

Recurrence is not statistically related to the presence of menarche at the first episode of torsion.

This multicenter study showed that oophorectomy is still a widely used procedure for OT despite the excellent results of conservative therapy reported in the literature in recent years. The percentage of $62 \%$ of oophorectomy ( 77 of 125 ) highlighted in this multicenter study must be taken into consideration and a greater awareness should support conservative therapy. Furthermore, despite the current widespread use of laparoscopic surgery, the open technique still represents more than $40 \%$ of the approaches used for OT. OPY does not adversely affect ultrasonographic followup at 1 year after surgery $(P=1.00)$. OT or retorsion after 


\section{OPY is a rare event (5.2\%; 1 of 19). Indeed, when possible OPY should be performed at the first episode of torsion to eliminate the not uncommon possibility of recurrence.}

\section{References}

1. Adelman S, Benson CD, Hertzler JH: Surgical lesions of the ovary in infancy and childhood. Surg Gynecol Obstet 1975; 141:219

2. Cass DL: Ovarian torsion. Semin Pediatr Surg 2005; 14:86

3. Beaunoyer M, Chapdelaine J, Bouchard S, et al: Asynchronous bilateral ovarian torsion. J Pediatr Surg 2004; 39:746

4. Kokoska ER, Keller MS, Weber TR: Acute ovarian torsion in children. Am J Surg 2000; 180:462

5. Emonts M, Doornewaard H, Admiraal JC: Adnexal torsion in very young girls: diagnostic pitfalls. Eur J Obstet Gynecol Reprod Biol 2004; 116:207

6. Spigland N, Ducharme JC, Yazbeck S: Adnexal torsion in children. J Pediatr Surg 1989; $24: 974$

7. Mordehai J, Mares AJ, Barki Y, et al: Torsion of uterine adnexa in neonates and children: a report of 20 cases. J Pediatr Surg 1991; 26:1195

8. Cass DL, Hawkins E, Brandt ML, et al: Surgery for ovarian masses in infants, children, and adolescents: 102 consecutive patients treated in a 15-year period. J Pediatr Surg 2001; 36:693

9. Aziz D, Davis V, Allen L, et al: Ovarian torsion in children: is oophorectomy necessary? J Pediatr Surg 2004; 39:750

10. Anders JF, Powell EC: Urgency of evaluation and outcome of acute ovarian torsion pediatric patients. Arch Pediatr Adolesc Med 2005; 159:532

11. Rody A, Jackisch C, Klockenbusch W, et al: Conservative management of adnexal torsion. A case-report and review of the literature. Eur J Obstet Gynecol Reprod Biol 2002; 101:83

12. Ozcan C, Celik A, Ozok G, et al: Adnexal torsion in children may have catastrophic sequel: asynchronous bilateral torsion. J Pediatr Surg 2002; 37:1617

13. Tsafrir Z, Azem F, Hasson J, et al: Risk factors, symptoms, and treatment of ovarian torsion in children: the twelve-year experience of one center. Minim Invasive Gynecol 2012; 19:29

14. Breech LL, Hillard PJ: Adnexal torsion in pediatric and adolescent girls. Curr Opin Obstet Gynecol 2005; 17:483

15. Bertozzi M, Riccioni S, Valoncelli $C$, et al: The diagnosis and management of ovarian cysts in children. J Pediatr Adolesc Gynecol 2013. http://dx.doi.org/10. 1016/j.jpag.2013.01.061, in press.

16. Crouch NS, Gyampoh B, Cutner AS, et al: Ovarian torsion: to pex or not to pex? Case report and review of the literature. J Pediatr Adolesc Gynecol 2003; 16:381

17. Svensson JF, Larsson A, Uusijarvi J, et al: Oophoropexy, hyperbaric oxygen therapy, and contrast-enhanced ultrasound after asynchronous bilatera ovarian torsion. J Pediatr Surg 2008; 43:1380

18. McCrea RS: Uterine adnexal torsion with subsequent contralateral recurrence. J Reprod Med 1980; 25:123
19. Guileyardo JM: Neonatal ovarian torsion. Am J Dis Child 1982; 136:945

20. Thorp JM Jr, Wells SR, Droegemueller W: Ovarian suspension in massive ovarian edema. Obstet Gynecol 1990; 76:912

21. Rollene N, Nunn M, Wilson $\mathrm{T}$, et al: Recurrent ovarian torsion in premenarchal adolescent girl: contemporary surgical management. Obstet Gynecol 2009; 114:422

22. Shadinger LL, Andreotti RF, Kurian RL: Preoperative sonographic and clinical characteristics as predictors of ovarian torsion. J Ultrasound Med 2008; 27:7

23. Rousseau V, Massicot R, Darwish AA, et al: Emergency management and conservative surgery of ovarian torsion in children: a report of 40 cases. J Pediatr Adolesc Gynecol 2008; 21:201

24. Servaes S, Zurakowski D, Laufer MR, et al: Sonographic findings of ovarian torsion in children. Pediatr Radiol 2007; 37:446

25. Dunnihoo DR, Wolff J: Bilateral torsion of the adnexa: a case report and a review of the world literature. Obstet Gynecol 1984; 64:55S

26. Anspach B: The torsion of tubal enlargements with special reference to pyosalpinx. Am J Obstet Gynecol 1912; 66:553

27. James DF, Barber HR, Graber EA: Torsion of normal uterine adnexa in children. Report of three cases. Obstet Gynecol 1970; 35:226

28. Evans JP: Torsion of the normal uterine adnexa in premenarchal girls. J Pediatr Surg 1978; 13:195

29. Galinier P, Carfagna L, Delsol M, et al: Ovarian torsion. Management and ovarian prognosis: a report of 45 cases. J Pediatr Surg 2009; 44:1759

30. Geimanaite L, Trainavicius K: Ovarian torsion in children: management and outcomes. J Pediatr Surg 2013; 48:1946

31. Bertozzi M, Magrini E, Bellucci C, et al: Recurrent ipsilateral ovarian torsion: case report and literature review. J Pediatr Adolesc Gynecol 2015; 28:e197

32. Smorgick N, Melcer Y, Sarig-Meth T, et al: High risk of recurrent torsion in premenarchal girls with torsion of normal adnexa. Fertil Steril 2016; 105:1561

33. Celik A, Ergun O, Aldemir $\mathrm{H}$, et al: Long-term results of conservative management of adnexal torsion in children. J Pediatr Surg 2005; 40:704

34. Abes M, Sarihan H: OPY in children with ovarian torsion. Eur J Pediatr Surg 2004; $14: 168$

35. Germain M, Rarick T, Robins E: Management of intermittent ovarian torsion by laparoscopic oophoropexy. Obstet Gynecol 1996; 88:715

36. Sheizaf B, Ohana E, Weintraub AY: "Habitual adnexal torsions"-recurrence after two oophoropexies in a prepubertal girl: a case report and review of the literature. J Pediatr Adolesc Gynecol 2013; 26:e81

37. Pansky M, Smorgick N, Herman A, et al: Torsion of normal adnexa in postmenarchal women and risk of recurrence. Obstet Gynecol 2007; 109:355

38. Fuchs N, Smorgick N, Tovbin Y, et al: Oophoropexy to prevent adnexal torsion: how, when, and for whom? J Minim Invasive Gynecol 2010; 17:205

39. Tsafrir Z, Hasson J, Levin I, et al: Adnexal torsion: cystectomy and ovarian fixation are equally important in preventing recurrence. Eur J Obstet Gynecol Reprod Biol 2012; 162:203

40. Simsek E, Kilicdag E, Kalayci $\mathrm{H}$, et al: Repeated ovariopexy failure in recurrent adnexal torsion: combined approach and review of the literature. Eur J Obstet Gynecol Reprod Biol 2013; 170:305 